\title{
SPECTRAL PROPERTIES OF RANDOM NON-SELF-ADJOINT MATRICES AND OPERATORS
}

\author{
E.B. Davies
}

February 2000

\begin{abstract}
We describe some numerical experiments which determine the degree of spectral instability of medium size randomly generated matrices which are far from self-adjoint. The conclusion is that the eigenvalues are likely to be intrinsically uncomputable for similar matrices of a larger size. We also describe a stochastic family of bounded operators in infinite dimensions for almost all of which the eigenvectors generate a dense linear subspace, but the eigenvalues do not determine the spectrum. Our results imply that the spectrum of the non-self-adjoint Anderson model changes suddenly as one passes to the infinite volume limit.
\end{abstract}

AMS subject classifications: 65F15, 65F22, 15A18, 15A52, 47A10, 47A75, 47B80, 60H25.

keywords: eigenvalues, spectral instability, matrices, computability, pseudospectrum, Schrödinger operator, Anderson model.

\section{Introduction}

In a series of recent papers [2,4-10] a number of authors have investigated the spectral properties of non-self-adjoint matrices and operators, coming to the conclusion that the eigenvalues are frequently highly unstable under small perturbations of the coefficients of the matrices. Trefethen has investigated a series of numerical examples using the concept of pseudospectrum (the contour plots of the resolvent norm), which provides a graphical demonstration of the degree of instability, [20, 21]. This concept is, however, not well adapted to the consideration of very large numbers of randomly generated matrices, for which one needs to produce a 
numerical measure of instability of the spectrum. In this paper we define such an instability index, and compute it for a series of randomly generated $N \times N$ matrices for various values of $N$ up to 50. Our numerical results are presented in Section 3 , following a short theoretical section which describes the concepts involved. Our results are fully in line with what would be expected by experts in pseudospectral theory, but we believe that such a systematic quantitative investigation of non-selfadjoint random matrices has not previously been carried out, and it is clear that much of the spectral theory community is not aware of these phenomena.

From Section 5 onwards we consider a related problem for a stochastic family of non-self-adjoint bounded operators in infinite volume, i.e acting on $l^{2}\left(\mathbf{Z}^{n}\right)$. For one example we prove that although the eigenvectors of almost all of the operators span a dense linear subspace, they do not form a basis, and the spectrum is much larger than the closure of the set of eigenvalues. Section 6 is devoted to spelling out the implications of our results for the non-self-adjoint Anderson model of HatanoNelson, which has been the focus of much recent attention. 12, 13, 14, 17 We find that the asymptotic behaviour of the eigenvalues as the volume increases does not describe the full spectrum of the infinite volume problem. The reason is that there are many approximate eigenvalues of the finite volume problem which are not close to true eigenvalues but which nevertheless affect the infinite volume limit. This situation is typical of non-self-adjoint operators, for which the eigenvectors need not be even approximately orthogonal.

\section{The Theoretical Context}

Throughout this section we suppose that $A$ is an $N \times N$ matrix with distinct eigenvalues. This is generically true, since the set of matrices with repeated eigenvalues forms a lower dimensional set with zero Lebesgue measure. All vectors below are assumed to be column vectors unless otherwise stated, and * denotes the conjugate transpose. Norms of vectors are always their Euclidean norms and norms of operators are always the corresponding operator norms.

Let $\lambda$ be an eigenvalue of $A$ of multiplicity 1, with corresponding eigenvector $\phi$. Let $\psi$ be an eigenvector of $A^{*}$ with eigenvalue $\mu$. If $\mu \neq \bar{\lambda}$ then $\psi^{*} \phi=0$ and otherwise this inner product is non-zero. Assuming that $\mu=\bar{\lambda}$, the spectral projection $P$ associated to the eigenvalue is given by

$$
P f=\left(\psi^{*} \phi\right)^{-1}\left(\psi^{*} f\right) \phi
$$

and its norm is

$$
\|P\|=\|\phi\|\|\psi\| /\left|\psi^{*} \phi\right|
$$

If we label all the above quantities by $n$ for $n=1, \ldots, N$, then we may define the 
instability index of $A$ by

$$
i(A)=\max \left\{\left\|P_{n}\right\|: n=1, \ldots, N\right\} .
$$

This is unrelated to its condition number

$$
\kappa(A)=\|A\|\left\|A^{-1}\right\|
$$

since the instability index of a self-adjoint or normal matrix always equals 1 , but its condition number may be arbitrarily large; in the converse direction the matrix

$$
\left(\begin{array}{cc}
1 & 1 \\
0 & 1+\delta
\end{array}\right)
$$

has small condition number but unbounded instability index as $\delta \rightarrow 0$. The norm of a particular spectral projection is also called the condition number of the eigenvalue, and is known to measure how unstable the eigenvalue is under small perturbations of the matrix, [1, 23, 24, [16, sect. 11.2]. We emphasize that if the norm of the spectral projection is very large the instability of the eigenvalue it intrinsic: it does not depend on the particular method of computing it. The norm of $P$ is always at least 1 and equals 1 if and only if $P$ is orthogonal, or equivalently if $\phi=\psi$. The following proposition relates this index to other measures of how far the matrix is from being normal, [1, 23, 24.

Proposition 1 We have the relations

$$
(i) \Rightarrow(i i) \Rightarrow(i i i) \Rightarrow(i v)
$$

between the following conditions, the constant $k$ being the same in all cases.

(i) There exists an invertible matrix $V$ with $\kappa(V) \leq k$ such that $D=V^{-1} A V$ is diagonal.

(ii) The functional calculus satisfies

$$
\|f(A)\| \leq k\|f\|_{\infty}
$$

for all complex-valued functions $f$ defined on $\operatorname{Spec}(A)$, where

$$
\|f\|_{\infty}=\max \{|f(\lambda)|: \lambda \in \operatorname{Spec}(A)\} .
$$

(iii) The resolvent operators satisfy

$$
\left\|(A-z I)^{-1}\right\| \leq k \operatorname{dist}(z, \operatorname{Spec}(A))^{-1}
$$

for all $z \notin \operatorname{Spec}(A)$, where dist is the Euclidean distance of a point from a set.

(iv) The spectral projection $P$ of every eigenvalue $\lambda$ of $A$ satisfies $\|P\| \leq k$, and hence

$$
i(A) \leq k
$$


$\underline{\text { Proof }}$

(i) $\Rightarrow$ (ii) We use $f(A)=V f(D) V^{-1}, \operatorname{Spec}(A)=\operatorname{Spec}(D)$ and

$$
\|f(D)\|=\|f\|_{\infty}
$$

(ii) $\Rightarrow$ (iii) This is a matter of considering the particular function $f(\lambda)=(\lambda-z)^{-1}$.

(iii) $\Rightarrow$ (iv) We express the spectral projection as a contour integral of resolvent operators around a small circle centred at $\lambda$.

Note 2 The proof of the theorem remains valid if we replace the use of the Euclidean norm on $\mathbf{C}^{N}$ by any other norm, provided the appropriate operator norm is used for matrices and the operator norm of any diagonal matrix $D$ is given by

$$
\|D\|=\max \left\{\left|D_{n, n}\right|: 1 \leq n \leq N\right\} .
$$

This is equivalent to the norm being absolute [16, sect. 10.5] and holds in particular for all of the $l^{p}$ norms. However, certain other matters, such as the identification of orthogonal projections with those of norm 1, are dependent on the use of the Euclidean norm.

Note 3 If we assume condition (iv) of the above theorem then it follows from the formula

$$
(A-z)^{-1}=\sum_{i} P_{i}\left(\lambda_{i}-z\right)^{-1}
$$

that

$$
\left\|(A-z)^{-1}\right\| \leq N k \operatorname{dist}(\operatorname{Spec}(A), z)^{-1}
$$

Since the value of $k$ frequently increases exponentially with the dimension $N, 20$, 25. one must expect pseudospectral information and that obtained from the instability index to be broadly equivalent.

The point of the theorem is that if any spectral projection of $A$ has very large norm, then the constant $k$ of any of the earlier conditions must be very large, and diagonalization of the matrix $A$ is an intrinsically ill-conditioned procedure.

There is a family of matrices for which the instability index defined above can be computed in closed form. This is of some interest for its own sake, but we used it to verify the algorithm used to compute the instability indices of randomly generated matrices. We assume that

$$
A_{m, n}=f(m-n) a^{m-n}
$$

where $a>1$ and $f: \mathbf{Z} \rightarrow \mathbf{C}$ is any function which is periodic with period $N$. 
Theorem 4 The eigenvectors of $A$ are of the form

$$
\phi_{r}(n)=a^{n} \mathrm{e}^{2 \pi i r n / N}
$$

where $r=1, \ldots, N$. The corresponding spectral projections all have norm

$$
c=\frac{a}{\left(a^{2}-1\right) N}\left(a^{N}-a^{-N}\right) .
$$

Thus the instability index of $A$ is also $c$.

Proof The first statement is a matter of applying the matrix to such a vector, and noting that the set of all such vectors is a basis for $\mathbf{C}^{N}$. The corresponding eigenvectors of $A^{*}$ are

$$
\psi_{r}(n)=a^{-n} \mathrm{e}^{2 \pi i r n / N} .
$$

From these facts it is now easy to calculate the condition numbers of each of the eigenvalues using (11).

\section{$3 \quad$ Numerical Results}

We have applied the above ideas to a series of randomly generated tridiagonal $N \times N$ matrices for various values of $N$. The matrices are of the form $A_{m, n}$ so that $A_{m, n}=0$ for $|m-n|>1$, the other coefficients being chosen randomly and independently. If $m-n=1$ the coefficients are chosen using a uniform distribution on $[0,1]$, if $m-n=0$ the coefficients are chosen using a uniform distribution on $[0,2]$, and if $m-n=-1$ the coefficients are chosen using a uniform distribution on $[0,3]$.

For each $A$ we used Matlab to obtain an invertible matrix $V$ and a diagonal matrix $D$ such that $V^{-1} A V=D$. The columns of $V$ are then the eigenvectors $\phi_{n}$ of $A$, and are provided by Matlab in normalized form. The rows of $V^{-1}$ are $\psi_{m}^{*}$ where $\psi_{n}$ are the eigenvectors of $A^{*}$. We get $\psi_{n}^{*} \phi_{n}=1$ automatically, so

$$
\left\|P_{n}\right\|^{2}=\left\|\psi_{n}\right\|^{2}=E_{n, n}
$$

where $E=V^{-1}\left(V^{-1}\right)^{*}$. We thus finally get

$$
i(A)=\max \left\{\left|E_{n, n}\right|^{1 / 2}: 1 \leq n \leq N\right\} .
$$

For each of $M$ randomly generated $N \times N$ matrices $A$ we computed the instability index using (2), and then sorted the data points into increasing order. Defining $\mathcal{P}_{r}$ to be the number such that such that $r \%$ of the instability indices were less than $\mathcal{P}_{r}$, we determined $\mathcal{P}_{50}$ and $\mathcal{P}_{95}$ for various values of $N$. In fact we carried 
out each computation twice in order to give some idea of the degree of reliability of our results; we tabulated the average of the two values and the difference $\mathcal{D}_{r}$ expressed as a proportion of the average.

\begin{tabular}{cccccccc}
$N$ & $M$ & $\mathcal{P}_{50}$ & $\mathcal{D}_{50}$ & $\mathcal{P}_{95}$ & $\mathcal{D}_{95}$ & $\log \left(\mathcal{P}_{50}\right) / N$ & $\log \left(\mathcal{P}_{95}\right) / N$ \\
\hline 10 & $10^{6}$ & 18.47 & 0.007 & 304.1 & 0.001 & 0.292 & 0.572 \\
20 & $10^{6}$ & 684.9 & 0.005 & $6.13 \times 10^{4}$ & 0.008 & 0.326 & 0.551 \\
30 & $4 \times 10^{5}$ & $2.138 \times 10^{4}$ & 0.013 & $7.48 \times 10^{6}$ & 0.001 & 0.332 & 0.528 \\
40 & $10^{5}$ & $5.77 \times 10^{5}$ & 0.047 & $6.68 \times 10^{8}$ & 0.050 & 0.332 & 0.508 \\
50 & $10^{5}$ & $1.43 \times 10^{7}$ & 0.016 & $4.60 \times 10^{10}$ & 0.032 & 0.330 & 0.491
\end{tabular}

It is clear from our results that both $\mathcal{P}_{50}$ and $\mathcal{P}_{95}$ increase extremely rapidly with $N$. In fact our results support the conjecture that $\mathcal{P}_{50} \sim \mathrm{e}^{N / 3}$ as $N \rightarrow \infty$. Matlab is already having a little difficulty in computing the eigenvalues of the matrices for $N=50$, and fails entirely for $N=200$. Such exponential increase has also been found by Trefethen et al for other models using pseudospectral methods. [20, 25]

An alternative approach to the above questions would be to compute the expected value of $i(A)$ over a large sample of matrices $A$, but this would have the disadvantage of being unduly influenced by the very large size of $i(A)$ for a small proportion of choices of $A$.

\section{Distribution of Norms of Spectral Projections}

Instead of studying $i(A)$ for randomly distributed matrices $A$, one may examine how the norms of the individual spectral projections are distributed. This may be done in several ways. In the first we sort the norms of the spectral projections of a particular $N \times N$ matrix $A$ in increasing order, but instead of examining the largest of these, namely $i(A)$, we evaluate the number $j(A)$ half way through the list. We did this for a series of $10^{5}$ randomly generated $30 \times 30$ matrices, and discovered that for $50 \%$ of these matrices $j(A) \leq 221.2$. The fact that this number is so much smaller than $i(A)$ under the corresponding conditions indicates that only a small proportion of the spectral projections of a typical random matrix $A$ have really large norms.

A second procedure is to consider all of the norms of the spectral projections of the $10^{5}$ randomly generated $30 \times 30$ matrices as one list of $30 \times 10^{5}$ numbers. When we carried out this numerical experiment we found that $50 \%$ of the norms so obtained were less than 157.8. There is no reason why the above two numbers should coincide, but they are of the same order of magnitude, leading to the same conclusion. 
The final and most interesting method is to carry out a spectral analysis of the covariance matrix associated with the norms of the spectral projections. We proceed as follows.

For each matrix $A$ we define the numbers $X_{n} \geq 0$ for $1 \leq n \leq N$ by

$$
X_{n}=\log \left(\left\|P_{n}\right\|\right)
$$

where these are sorted in increasing order. (It is possible to carry out similar calculations without taking the logarithm above, but the results are less compelling.) As $A$ varies within the usual class these provide a family of $N$ non-negative random variables whose covariance matrix is defined by

$$
C_{m, n}=\mathbf{E}\left[X_{m} X_{n}\right] .
$$

The eigenvalues and eigenvectors of this matrix provide information about the distribution of the norms of the spectral projections of 'typical' random matrices.

We carried out the above computation for a sample of $10^{5}$ randomly distributed $10 \times$ 10 matrices. The eigenvalues of $C$ were found to be $0.0060,0.0196,0.0239,0.0323$, $0.0433,0.0613,0.1034,0.2133,0.7169,54.6547$. The fact that one eigenvalue is so dominant is very striking, and indicates that to a very good approximation most of the random matrices have very similar distributions of the norms of their spectral projections. We repeated the computation for a sample of $10^{5}$ randomly distributed $30 \times 30$ matrices. There were only 4 eigenvalues larger than 1 , these being $1.5,3.1,12.02,1322.9$.

In both cases we computed the eigenvector $v$ corresponding to the largest eigenvalue of the covariance matrix $C$. We found that $v_{n}$ was close to being proportional to $n$. This corresponds to the norms $\left\|P_{n}\right\|$ of a 'typical' random matrix $A$ increasing exponentially with $n$. We conjecture that the dominance of the leading eigenvalue increases and the rate of increase of the spectral norms becomes more accurately exponential as the size $N$ of the matrices increases.

Let us be more precise about this. Given $N$ we consider the sample space

$$
\Omega_{N}=[0,1]^{N-1} \times[0,2]^{N} \times[0,3]^{N-1}
$$

provided with the uniform probability distribution. For each $\omega \in \Omega_{N}$ we described how to construct a matrix $A_{\omega}$ and then the $N$ random variables $X_{n}$ defined by (3). These have a symmetric $N \times N$ covariance matrix $C$ whose eigenvalues may be ordered so as to satisfy

$$
0 \leq \lambda_{1} \leq \ldots \leq \lambda_{N}
$$

We finally define

$$
\mu_{N}=\frac{\lambda_{N-1}}{\lambda_{N}}
$$


making explicit the dependence of $\mu$ on $N$. The conjecture is then that

$$
\lim _{N \rightarrow \infty} \mu_{N}=0 .
$$

We tested this hypothesis by considering a series of $T$ randomly generated $N \times N$ matrices for various values of $T$ and $N$. The results below provide some support for the conjecture.

\begin{tabular}{ccccc}
$N$ & $T$ & $\lambda_{N-1}$ & $\lambda_{N}$ & $\mu_{N}$ \\
\hline 10 & $10^{6}$ & 0.725 & 54.99 & 0.0132 \\
20 & $10^{6}$ & 4.422 & 422.40 & 0.0105 \\
30 & $4 \times 10^{5}$ & 12.02 & 1319.5 & 0.0091 \\
40 & $10^{5}$ & 24.15 & 2928.4 & 0.0082 \\
50 & $10^{5}$ & 40.73 & 5408.9 & 0.0075
\end{tabular}

\section{Operators with Randomly Distributed Coeffi- cients}

In this section we present some ideas relating to a random family of bounded linear operators acting on the infinite-dimensional Hilbert space $l^{2}(\mathbf{Z})$. This may be regarded as the infinite volume limit of our earlier problems, although pseudospectral theory suggests that one should also study the 'same' operator on $l^{2}\left(\mathbf{Z}^{+}\right)$. Physically the choice between these two operators depends upon whether one wishes to include end effects, which are present both for large finite intervals and for the operator on $l^{2}\left(\mathbf{Z}^{+}\right)$. Our methods can easily be adapted to this case, but we do not spell out the modifications needed.

We first formulate the ideas at a moderately general level, and only later restrict attention to the non-self-adjoint Anderson model of Hatano-Nelson. Let $A$ be the operator associated with an infinite matrix $\left\{A_{m, n}\right\}$ where $A_{m, n}=0$ if $|m-n|>$ 1 ; we suppose that the vectors $v_{n}=\left(A_{n+1, n}, A_{n, n}, A_{n, n+1}\right) \in \mathbf{C}^{3}$ are distributed independently according to a common law $\mu$, where $\mu$ is a probability measure on $\mathbf{C}^{3}$ with compact support $K$. It follows from the assumptions and the fact that the matrix $\left\{A_{m, n}\right\}$ is tridiagonal that it is associated with a bounded operator $A$ such that

$$
\|A\| \leq \sum_{r=1}^{3} \max \left\{\left|w_{r}\right|: w \in K\right\} .
$$

The above procedure defines a stochastic family of bounded operators $A_{\omega}$, for $\omega$ in the sample space $K^{\infty}$. It is of some interest that the results which we obtain are not truly probabilistic: the statements of our theorems only involve the set $K$ rather than the probability measure $\mu$. 
In order to prove some results about the spectra of operators in the family we introduce a notion from [11]. Given any bounded operator $X$ on a Hilbert space $\mathcal{H}$, we say that the operator $Y$ lies in its limit class, $Y \in \mathcal{C}(X)$, if there exists a sequence $U_{s}$ of unitary operators on $\mathcal{H}$ such that $U_{s}^{*} X U_{s}$ converges strongly to $Y$ as $s \rightarrow \infty$. We also define the approximate point spectrum $\sigma(X)$ of $X$ to be the set of all $\lambda \in \mathbf{C}$ for which there exists a sequence of vectors $f_{s} \in \mathcal{H}$ such that $\left\|f_{s}\right\|=1$ and

$$
\lim _{s \rightarrow \infty}\left\|X f_{s}-\lambda f_{s}\right\|=0 .
$$

In all the examples in this paper $\sigma(X)=\operatorname{Spec}(X)$ almost surely but it seems desirable for the sake of possible future applications to keep the logical distinction. The following two lemmas are modifications of a classical result of Pastur stating that the spectrum of random tridiagonal operators is almost surely constant; the proof uses translation ergodicity of the class of operators. [4, p 167]

Lemma 5 If $Y$ lies in the limit class of $X$ then $\sigma(X) \supseteq \sigma(Y)$. Hence

$$
\operatorname{Spec}(X) \supseteq \bigcup\{\sigma(Y): Y \in \mathcal{C}(X)\}
$$

In particular if each lies in the limit class of the other then $\sigma(X)=\sigma(Y)$.

$\underline{\text { Proof }}$ Suppose that for some $\lambda \in \mathbf{C}$ and all $\varepsilon>0$ there exists $f \in \mathcal{H}$ such that $\|f\|=1$ and $\|Y f-\lambda f\|<\varepsilon$. Now put $f_{s}=U_{s} f$ and observe that

$$
\left\|X f_{s}-\lambda f_{s}\right\|=\left\|U_{s}^{*} X U_{s} f-\lambda f\right\| \rightarrow\|Y f-\lambda f\|
$$

as $s \rightarrow \infty$. Therefore $\left\|X f_{s}-\lambda f_{s}\right\|<\varepsilon$ for all large enough $s$, and $\lambda \in \sigma(X)$.

We apply the above to the randomly generated operators $A_{\omega}$ acting on $l^{2}(\mathbf{Z})$.

Lemma 6 The limit class of almost every operator $A_{\omega}$ generated as described contains every operator $A_{\tilde{\omega}}$ such that $\tilde{\omega} \in K^{\infty}$.

Proof Let $N \in \mathbf{Z}^{+}$and $\tilde{\omega}=\left\{\tilde{v}_{n}\right\}_{n \in \mathbf{Z}} \in K^{\infty}$. Given $\varepsilon>0$ we put

$$
V_{n}=\left\{z \in \mathbf{C}^{3}:\left\|\tilde{v}_{n}-z\right\|<\varepsilon\right\}
$$

so that $\mu\left(V_{n}\right)>0$ for all $n$. Taking $U_{s}: l^{2}(\mathbf{Z}) \rightarrow l^{2}(Z)$ to be the unitary operators associated with appropriate translations of $\mathbf{Z}$, we need to show that for almost every $\omega=\left\{v_{n}\right\} \in K^{\infty}$ there exists $M$ such that $v_{n+M} \in V_{n}$ for all $-N \leq n \leq N$.

To prove this we put $V=\prod_{n=-N}^{N} V_{n}$ and

$$
\omega=\left\{v_{n}\right\}_{n \in \mathbf{Z}}=\left\{w_{m}\right\}_{m \in \mathbf{Z}}
$$

where

$$
w_{m}=\left\{v_{m(2 N+1)+r}\right\}_{r=-N}^{N} \in K^{2 N+1} .
$$


The vectors $w_{m}$ are independent and identically distributed with positive probability that $w_{m} \in V$. Hence the probability that none of the $w_{m}$ lie in $V$ is zero.

As an application of the lemma, let $v, w \in C^{3}$ and let $B_{v, w}$ be the bounded operator associated with the infinite matrix $\left\{B_{m, n}\right\}$ such that $B_{m, n}=0$ if $|m-n|>1$; we also assume that the vector $\left(B_{n+1, n}, B_{n, n}, B_{n, n+1}\right)$ equals $v$ if $n \geq 1$ and equals $w$ if $n \leq 0$. The point of introducing this class of operators is that they are similar to operators whose spectrum is well understood. [Q], [3], [15]

Corollary 7 With probability 1 one has

$$
\operatorname{Spec}\left(A_{\omega}\right) \supseteq \bigcup\left\{\sigma\left(B_{v, w}\right): v, w \in K\right\} .
$$

Proof Every operator $B_{v, w}$ lies in the limit class of $A$ by Lemma 5 .

The significance of the above lemmas is best seen by applying them to an example. We assume that $\mu$ is a probability measure concentrated on a finite subset $F$ of $\mathbf{R}^{2}$ (more general probability measures can also be treated). We assume that $\mu(0,0)>0$ and that any other point $(x, y)$ with $\mu(x, y)>0$ satisfies $x>0$ and $y>0$. We then define the random family of operators $A$ on $l^{2}(\mathbf{Z})$ as described above with the following simplification: we assume that all $A_{n, n}=0$ and that the vectors $\left(A_{n+1, n}, A_{n, n+1}\right)$ are distributed independently according to the law of $\mu$.

Theorem 8 Depending to the choice of $\mu$, either $A_{\omega}$ is self-adjoint with probability 1 or it is non-self-adjoint with probability 1 . In the latter case $A_{\omega}$ possesses a countable set of eigenvalues whose corresponding eigenvectors span a dense linear subspace of $l^{2}(\mathbf{Z})$. With probability 1 the set of eigenvectors is not a basis of $l^{2}(\mathbf{Z})$.

Proof The self-adjoint case occurs when the support of $\mu$ is contained in the diagonal set $\left\{(x, y) \in \mathbf{R}^{2}: x=y\right\}$ and we assume that this is not the case below.

With probability 1 an infinite number of the pairs $\left(A_{n+1, n}, A_{n, n+1}\right)$ are equal to $(0,0)$, and we assume that this happens for the increasing sequence $\left\{N_{r}\right\}$ where $r \in \mathbf{Z}$. It may then be seen that $A_{\omega}$ can be decomposed as the orthogonal direct sum of matrices $C_{r}$ of sizes $M_{r} \times M_{r}$ where $M_{r}=N_{r}-N_{r-1}$. Since each matrix $C_{r}$ is tridiagonal with positive off-diagonal entries and zero diagonal entries, its eigenvalues are all real and of multiplicity one. By combining all of the eigenvectors of the $C_{r}$ as $r$ increases we see that the eigenvectors of $A_{\omega}$ almost surely span a dense linear subspace of $l^{2}(\mathbf{Z})$. It remains only to prove that this set of eigenvectors is almost surely not a basis.

Let $(x, y)$ be any point in $\mathbf{R}^{2}$ with $0<x<y$ and $\mu(x, y)>0$. Since the pairs of coefficients of $A_{\omega}$ are chosen independently, among the $C_{r}$ there almost surely 
exist all $s \times s$ matrices of the form $B_{s}$ where

$$
B_{s}= \begin{cases}x & \text { if } m=n+1 \\ y & \text { if } m=n-1 \\ 0 & \text { otherwise }\end{cases}
$$

Now the eigenvectors of each $B_{s}$ are given explicitly by

$$
\phi_{k, r}=\sin \left(\frac{\pi k r}{s+1}\right)\left(\frac{x}{y}\right)^{r / 2}
$$

and the corresponding eigenvectors of the adjoint operator are

$$
\psi_{k, r}=\sin \left(\frac{\pi k r}{s+1}\right)\left(\frac{y}{x}\right)^{r / 2}
$$

where $k$ labels which eigenvector is being considered and $r$ which coefficient. It follows that

$$
\begin{aligned}
\left\langle\phi_{k}, \psi_{k}\right\rangle & =\sum_{r=1}^{s} \sin \left(\frac{\pi k r}{s+1}\right)^{2} \\
\left\|\phi_{k}\right\|^{2} & =\sum_{r=1}^{s} \sin \left(\frac{\pi k r}{s+1}\right)^{2}\left|\frac{x}{y}\right|^{r} \\
\left\|\psi_{k}\right\|^{2} & =\sum_{r=1}^{s} \sin \left(\frac{\pi k r}{s+1}\right)^{2}\left|\frac{y}{x}\right|^{r}
\end{aligned}
$$

An application of (11) now shows that $\lim _{s \rightarrow \infty} i\left(B_{s}\right)=+\infty$, from which it follows that with probability 1 the norms of the spectral projections of $A_{\omega}$ are not uniformly bounded. This implies that the eigenvectors cannot form a basis.

An example of Zabzyk which has some similarities to those of the above theorem is discussed in Theorem 2.17 of [5], where the failure of the spectral mapping theorem for semigroups is demonstrated. Another type of example involving differential operators whose eigenvectors do not form a basis was presented in [8, 9, [10]. It appears that such a situation is relatively common for non-self-adjoint operators in infinite dimensions. Since the eigenvectors do not form a basis for the Hilbert space, there is no reason to expect that they determine the spectral behaviour of $A_{\omega}$. We continue with the hypotheses formulated before Theorem 7 .

Theorem 9 The eigenvalues of the operator $A_{\omega}$ are almost surely all real. However the spectrum of $A_{\omega}$ almost surely contains the interior of the ellipse E defined by

$$
E=\left\{x \mathrm{e}^{i \theta}+y \mathrm{e}^{-i \theta}: \theta \in[0,2 \pi]\right\}
$$

for every non-zero $(x, y) \in F$. 
Proof The operator $A_{\omega}$ is almost surely the orthogonal direct sum of tridiagonal matrices $C_{r}$, where we continue to use our previous notation. Each $C_{r}$ is similar to a real symmetric matrix and therefore has real eigenvalues.

If $v=(x, y)$ is a non-zero point in $F$ then by Corollary 6 the spectrum of $A_{\omega}$ almost surely contains $\sigma\left(B_{0, v}\right)$. We assume that $0<x<y$, the case $0<y<x$ having a similar analysis involving the adjoint operators. A direct computation shows that if $z$ lies inside the said ellipse then both solutions $w_{i}$ of

$$
x-z w+y w^{2}=0
$$

satisfy $\left|w_{i}\right|<1$. It follows that $z$ is an eigenvalue of $B_{0, v}$, the eigenvector being the sequence $f \in l^{2}(\mathbf{Z})$ given by

$$
f_{r}=\left\{\begin{array}{cc}
w_{1}^{r}-w_{2}^{r} & \text { if } r \geq 1 \\
0 & \text { otherwise. }
\end{array}\right.
$$

\section{The Non-Self-Adjoint Anderson Model}

In this section we consider non-self-adjoint Anderson-type operators of the form

$$
H f_{n}=\mathrm{e}^{-g} f_{n-1}+\mathrm{e}^{g} f_{n+1}+V_{n} f_{n}
$$

where $g>0$ and $V$ is a random real-valued potential. We assume that the values of $V$ at different points are independent and identically distributed according to a probability law $\mu$ which has compact support $M \subseteq \mathbf{R}$.

A considerable amount of attention has already been paid to such operators, which arise in population biology and solid state physics, as cited in 112, 13, 14, 17. If one supposes that the operator acts on $l^{2}\{-N, N\}$ subject to Dirichlet boundary conditions then its spectrum is almost surely real, because $H$ is similar to the operator defined by the same formula but with $g=0$. The similarity is determined by the operator

$$
S f_{n}=\mathrm{e}^{n g} f_{n}
$$

which is bounded on this finite-dimensional space. On the other hand if one imposes periodic boundary conditions, then the spectrum of $H$ is much more interesting and has been analyzed in great detail in the limit of large $N$ both numerically and theoretically. [12, 13, 14, 17] The imposition of periodic boundary conditions was justified in Nelson-Shnerb by the fact that they were considering a rotationally invariant problem (the diffusion of bacteria in a rotating nutrient). Goldsheid has observed that the spectral results proved in [12 for periodic boundary conditions are equally valid for a wide range of quasi-periodic boundary conditions. However, Trefethen has pointed out that the spectral behaviour of this type of operator 
is highly problematical. 22 This phenomenon has been investigated from several points of view over the last decade, [2, 3, 7, 18, 19, 20, 21] and among the conclusions is the warning that one cannot assume that a solution of a non-linear equation is stable simply because the eigenvalues of its linearization about the solution have negative real parts.

We show that if one considers the non-self-adjoint Anderson model $H$ acting on $l^{2}(\mathbf{Z})$, the spectrum is entirely different from what one obtains by letting $N \rightarrow \infty$ subject to any of the above types of boundary condition. The following theorems are directed towards locating the spectrum of $H$, but it would clearly be highly desirable to find a precise formula for it.

Theorem 10 Let $H$ be defined on $l^{2}(\mathbf{Z})$ by (4) where $V$ satisfies the stated conditions. Then

$$
\operatorname{Spec}(H) \subseteq \operatorname{conv}(E)+\operatorname{conv}(M)
$$

almost surely, where conv denotes convex hull and $E$ is the ellipse

$$
E=\left\{\mathrm{e}^{g+i \theta}+\mathrm{e}^{-g-i \theta}: \theta \in[0,2 \pi]\right\}
$$

Proof Let $A$ be the operator obtained from $H$ by deleting the potential $V$, so that $\operatorname{Spec}(A)=E$. Then the result follows from the equation

$$
\operatorname{Spec}(H) \subseteq \operatorname{Num}(H) \subseteq \operatorname{Num}(A)+\operatorname{Num}(V)=\operatorname{conv}(E)+\operatorname{conv}(M) .
$$

where Num stands for the numerical range

$$
\operatorname{Num}(A)=\{\langle A f, f\rangle:\|f\|=1\} .
$$

Theorem 11 Under the above assumptions we also have

$$
\operatorname{Spec}(H) \subseteq\{z \in \mathbf{C}: \operatorname{dist}(z, E) \leq m\}
$$

almost surely, where dist is the distance function and

$$
m=\max \{|s|: s \in M\} .
$$

$\underline{\text { Proof }}$ Since $A$ is a normal operator with spectrum equal to $E$, we have

$$
\left\|(z-A)^{-1}\right\|=\operatorname{dist}(z, E)^{-1}
$$

for all $z \notin E$. Also $\|V\|=m$ almost surely, and the result follows by examining the convergence of the perturbation series

$$
(z-A-V)^{-1}=\sum_{n=0}^{\infty}(z-A)^{-1}\left(V(z-A)^{-1}\right)^{n} .
$$

In the reverse direction we may apply Lemma 4 and Theorem 5 to obtain the following result, which is further improved in Theorem 16. 
Theorem 12 With probability one, the spectrum of $H$ contains the set $E+m$ for every $m \in M$.

Proof The operator $A+m I$ lies in the limit class of $H$ for all $m \in M$ and its approximate point spectrum is $E+m$ (as is its spectrum).

Example 13 Let $\mu$, the measure determining $V$, have support $[-B, B]$. By applying Theorems 10 and 12 it follows that the spectrum of $H$ is almost surely equal to the convex set $E+[-B, B]$ provided $B \geq \mathrm{e}^{g}+\mathrm{e}^{-g}$. This is quite different from what occurs for the same problem on $l^{2}(-N, N)$ in the limit $N \rightarrow \infty$, whatever boundary conditions are assumed. The point is that for such operators approximate eigenvalues need not be close to genuine eigenvalues, and the full spectral behaviour of the operators can best be seen using pseudospectral theory.

The determination of the spectrum for smaller values of $B$ is more complicated. An application of Theorem 11 shows that if $B<\mathrm{e}^{g}-\mathrm{e}^{-g}$ then

$$
\operatorname{Spec}(H) \cap\{z \in \mathbf{C}:|z|<r\}=\emptyset
$$

where $r=\mathrm{e}^{g}-\mathrm{e}^{-g}-B>0$. Since $E \subseteq \operatorname{Spec}(H)$, it follows that the spectrum of $H$ has a hole in it.

We have not been able to determine the precise range of values of $g, B$ for which the spectrum contains a hole. A numerical solution seems out of the question because the spectrum is heavily affected by extremely infrequent 'regular structure' in the potentials.

\section{Classification of the Spectrum}

The classification of the spectrum of non-self-adjoint operators is in a primitive state by comparison with that of self-adjoint operators. We start at an abstract level.

If $f \in l^{2}(\mathbf{Z})$ satisfies $\|f\|=1$, we put

$$
\operatorname{var}(f)=\left\langle Q^{2} f, f\right\rangle-\langle Q f, f\rangle^{2}
$$

where $Q$ is the position operator, provided this is finite. If $A$ is a bounded linear

operator acting on $l^{2}(\mathbf{Z})$, we say that $\lambda$ lies in its localized spectrum $\sigma_{\text {loc }}(A)$ if there exists $c$ and a sequence $f_{n} \in l^{2}(\mathbf{Z})$ such that $\left\|f_{n}\right\|=1$ and $\operatorname{var}\left(f_{n}\right) \leq c$ for all $n$ and

$$
\lim _{n \rightarrow \infty}\left\|A f_{n}-\lambda f_{n}\right\|=0 .
$$

A more general definition is possible but not necessary for our purposes. The localized spectrum of $A$ includes all eigenvalues whose associated eigenvectors have 
finite variances, but need not be a closed set. We say that $B$ lies in the translation limit class of $A$ if there exists a sequence of unitary translation operators $U_{n}$ such that $U_{n}^{*} A U_{n}$ converges strongly to $B$ as $n \rightarrow \infty$.

Lemma 14 If $B$ lies in the translation limit class of $A$ then $\sigma_{\mathrm{loc}}(A) \supseteq \sigma_{\mathrm{loc}}(B)$. In particular $\lambda \in \sigma_{\mathrm{loc}}(A)$ for every eigenvalue $\lambda$ of $B$ whose corresponding eigenvector has finite variance.

Proof This is the same as that of Lemma 5, with the extra observation that the variance is unchanged by translations.

The following theorem establishes that the localized spectrum is distinct from the approximate point spectrum. We say that an operator $A$ acting on $l^{2}$ is a finite order convolution operator if it is of the form $A f=k * f$ where $*$ denotes convolution and $k$ has finite support.

Theorem 15 If $H=A+B$ where $A$ is a finite order convolution operator and $B$ is compact, then its localized spectrum is the set of all its eigenvalues whose corresponding eigenvectors have finite variance. If $B=0$ this set is empty.

Proof Let $\lambda \in \sigma_{\text {loc }}(H)$ and let $f_{n}$ satisfy $\left\|f_{n}\right\|=1, \operatorname{var}\left(f_{n}\right) \leq c$ and $\left\|H f_{n}-\lambda f_{n}\right\| \rightarrow$ 0 as $n \rightarrow \infty$. If we put $a_{n}=\left\langle Q f_{n}, f_{n}\right\rangle$ then we have two cases to consider.

If $a_{n}$ is an unbounded sequence then by passing to a subsequence we may assume that $a_{n}$ diverges. If we define $U_{n}$ by

$$
\left(U_{n} \phi\right)_{m}=\phi_{m-a_{n}}
$$

and put $g_{n}=U_{n}^{*} f_{n}$ then $\left\langle Q g_{n}, g_{n}\right\rangle=0$ and $\operatorname{var}\left(g_{n}\right)=\operatorname{var}\left(f_{n}\right)$. Hence $g_{n}$ lies in the compact set

$$
S=\left\{\phi \in l^{2}(\mathbf{Z}):\|\phi\|=1 \text { and }\left\langle Q^{2} \phi, \phi\right\rangle \leq c\right\}
$$

for all $n$. By passing to a convergent subsequence we assume further that $g_{n}$ converges to a limit $g \in S$. Now we also have

$$
\lim _{n \rightarrow \infty}\left\|U_{n}^{*} H U_{n} g_{n}-\lambda g_{n}\right\|=0
$$

where $U_{n}^{*} H U_{n}$ converges strongly to $A$ as $n \rightarrow \infty$. Hence $A g=\lambda g$, which contradicts the fact, proved using Fourier analysis, that the point spectrum of $A$ is empty.

The alternative case is that there exists $a$ such that $\left|a_{n}\right| \leq a$ for all $n$. It then follows that $f_{n}$ lies in the compact set

$$
T=\left\{\phi \in l^{2}(\mathbf{Z}):\|\phi\|=1 \text { and }\left\langle Q^{2} \phi, \phi\right\rangle \leq c+a^{2}\right\}
$$


for all $n$. By passing to a convergent subsequence we assume further that $f_{n}$ converges to a limit $f \in T$. It is now immediate that $H f=\lambda f$.

The final special case of the theorem follows from the fact that $A$ has empty point spectrum.

In spite of the above, in the following context the localized spectrum is quite different from the point spectrum. We place ourselves in the situation described in the first paragraph of Section 6. Using the formula (5) for the ellipse $E$, we define $I_{z}$ to be the open interior of $E+z$ and $O_{z}$ to be the open exterior of $E+z$ for any $z \in \mathbf{C}$.

Theorem 16 If $H$ is defined by (4) then

$$
\sigma_{\mathrm{loc}}(H) \supseteq \bigcup_{\alpha, \beta \in M}\left\{I_{\alpha} \cap O_{\beta}\right\}
$$

almost surely.

$\underline{\text { Proof }}$ Given $\alpha, \beta \in M$ the operator $K$ defined by

$$
(K f)_{n}= \begin{cases}\mathrm{e}^{-g} f_{n-1}+\alpha f_{n}+\mathrm{e}^{g} f_{n+1} & \text { if } n \geq 1 \\ \mathrm{e}^{-g} f_{n-1}+\beta f_{n}+\mathrm{e}^{g} f_{n+1} & \text { if } n \leq 0\end{cases}
$$

lies in the translation limit class of $H$ by Lemma 5 , so it is sufficient to prove that if $\lambda \in I_{\alpha} \cap O_{\beta}$ then $\lambda$ is an eigenvalue of $K$ whose corresponding eigenvector has finite variance.

The eigenvalues and eigenvectors of $K$ may be determined explicitly. For $n \geq 2$ we solve the equation

$$
\mathrm{e}^{-g} u^{-1}+\alpha+\mathrm{e}^{g} u=\lambda
$$

and observe using the Residue Theorem that $\lambda \in I_{\alpha}$ implies that there are two solutions $u_{1}, u_{2}$ which both satisfy $\left|u_{i}\right|<1$. For $n<0$ we solve

$$
\mathrm{e}^{-g} v^{-1}+\beta+\mathrm{e}^{g} v=\lambda
$$

and observe similarly that $\lambda \in O_{\beta}$ implies that there is a solution $v$ which satisfies $|v|>1$. We now define

$$
f_{n}=\left\{\begin{array}{cc}
v^{n} & \text { if } n \leq 0 \\
c_{1} u_{1}^{n}+c_{2} u_{2}^{n} & \text { if } n \leq 1
\end{array}\right.
$$

By an appropriate choice of $c_{1}, c_{2}$ we can ensure that $K f=\lambda f$ as required. 


\section{Higher Dimensions}

The results of the last sections can all be extended to higher dimensions, and we briefly describe the situation in $l^{2}\left(\mathbf{Z}^{2}\right)$. We assume that

$$
H f_{m, n}=\mathrm{e}^{-g} f_{m-1, n}+\mathrm{e}^{g} f_{m+1, n}+\mathrm{e}^{-h} f_{m, n-1}+\mathrm{e}^{h} f_{m, n+1}+V_{m, n} f_{m, n}
$$

where $g>0, h>0$ and $V$ is a random real-valued potential. We assume that $V$ has independent values at different points and that they are distributed according to a probability measure $\mu$ with compact support $M \subseteq \mathbf{R}$. If $V=0$ then Fourier analysis shows that

$$
\operatorname{Spec}(H)=E+F
$$

where $E, F$ are the ellipses

$$
E=\left\{\mathrm{e}^{g+i \theta}+\mathrm{e}^{-g-i \theta}: \theta \in[0,2 \pi]\right\}
$$

and

$$
F=\left\{\mathrm{e}^{h+i \theta}+\mathrm{e}^{-h-i \theta}: \theta \in[0,2 \pi]\right\} .
$$

It is routine to show that Theorem 12 has the analogue

Theorem 17 With probability one, the spectrum of $H$ contains the set $E+F+m$ for every $m \in M$.

Localization of the spectrum is more complicated but the following ideas provide some information. Let $A, B$ be two bounded real-valued functions defined on $\mathbf{Z}$ and define

$$
(A+B)_{m, n}=A_{m}+B_{n}
$$

Theorem 18 If $A+B$ lies in the translation limit class of $V$ almost surely, then

$$
\sigma_{\mathrm{loc}}(H) \supseteq \sigma_{\mathrm{loc}}\left(H_{1}\right)+\sigma_{\mathrm{loc}}\left(H_{2}\right)
$$

almost surely, where $H_{j}$ act on $l^{2}(\mathbf{Z})$ according to

$$
H_{1} f_{n}=\mathrm{e}^{-g} f_{n-1}+\mathrm{e}^{g} f_{n+1}+A_{n} f_{n}
$$

and

$$
H_{2} f_{n}=\mathrm{e}^{-h} f_{n-1}+\mathrm{e}^{h} f_{n+1}+B_{n} f_{n} .
$$


$\underline{\text { Proof }}$ We first observe that $H_{1} \otimes I+I \otimes H_{2}$ lies in the translation limit class of $H$ almost surely, so

$$
\sigma_{\text {loc }}(H) \supseteq \sigma_{\text {loc }}\left(H_{1} \otimes I+I \otimes H_{2}\right) .
$$

By considering test functions of the form $f_{1} \otimes f_{2}$ we also see that

$$
\sigma_{\mathrm{loc}}\left(H_{1} \otimes I+I \otimes H_{2}\right) \supseteq \sigma_{\mathrm{loc}}\left(H_{1}\right)+\sigma_{\mathrm{loc}}\left(H_{2}\right) .
$$

It might seem that the hypothesis of this theorem is rather special. However if the support $M$ of $\mu$ is an interval then the theorem is of real value. Under these assumptions if $\alpha, \beta \in M$ then $A+B$ lies in the translation limit class of $V$ almost surely, where

$$
A_{m}=B_{m}= \begin{cases}\alpha / 2 & \text { if } m \geq 0 \\ \beta / 2 & \text { otherwise. }\end{cases}
$$

The localized spectrum of such operators is considered in Theorem 16.

\section{Conclusions}

We have analyzed the spectral behaviour of a family of randomly generated nonself-adjoint matrices by a variety of different methods. Our conclusion is that their eigenvalues depend very sensitively on the matrix entries even for quite small matrix sizes. The standard proofs of the existence of the eigenvalues depend upon the fundamental theorem of algebra, of which there are many proofs, but it is clear that the well-known instability of the roots of high degree polynomials leads to the large value of the instability index of general non-self-adjoint matrices. This problem does not occur for self-adjoint matrices because the variational theorem implies that the eigenvalues of such matrices do not change much under small perturbations, [6]. Our results indicate that nothing of a comparable nature is likely to be available in the non-self-adjoint case.

We have also investigated a family of randomly generated non-self-adjoint bounded operators acting on an infinite-dimensional Hilbert space, for which the eigenvectors almost surely generate a dense linear subspace. In spite of this the eigenvectors almost surely do not form a basis and the eigenvalues almost surely generate only a small part of the spectrum. We finally proved that the full spectrum of the infinite

volume non-self-adjoint Anderson model bears little relationship with the infinite volume limit of the spectra of the same operator in finite intervals. 
Acknowledgments I would like to thank L N Trefethen, M Embree and I Goldsheid for valuable comments.

\section{References}

[1] Aslanyan A, Davies E B: On eigenfunction approximations for typical non-selfadjoint Schrödinger operators. Preprint, April 1999. Proc. Roy. Soc. London Ser. A. to appear.

[2] Böttcher A: Pseudospectra and singular values of large convolution operators. J. Int. Eqns. Appl. 6 (1994) 267-301.

[3] Böttcher A: Infinite matrices and projection methods. In Lectures on Operator Theory and its Applications (ed. Peter Lancaster), pp 2-74. Fields Institute Monographs, Amer. Math. Soc. Publ., Providence, RI., 1995.

[4] Cycon H L, Froese R G, Kirsch W, Simon B: Schrödinger Operators. SpringerVerlag, Berlin 1987.

[5] Davies E B: One-Parameter Semigroups. Academic Press, London, 1980.

[6] Davies E B: Spectral Theory and Differential Operators. Camb. Univ. Press, Cambridge, 1995.

[7] Davies E B: Pseudospectra of differential operators. J. Operator Theory 43 (2000) to appear.

[8] Davies E B: Pseudospectra, the harmonic oscillator and complex resonances. Proc. R. Soc. Lond. A, 455 (1999) 585-599.

[9] Davies E B: Semi-classical states for non-self-adjoint Schrödinger operators. Commun. Math. Phys. 200 (1999) 35-41.

[10] Davies E B: Wild spectral behaviour of anharmonic oscillators. Bull. London Math. Soc. (2000) to appear.

[11] Davies E B, Simon B: $L^{1}$ properties of intrinsic Schrödinger operators. J. Functional Anal. 65 (1986) 126-146.

[12] Goldsheid I Y, Khoruzhenko B A: Distribution of eigenvalues in non-Hermitian Anderson model. Phys. Rev. Lett. 80 (1998) 2897-2901.

[13] Hatano N, Nelson D R: Vortex pinning and non-Hermitian quantum mechanics. Phys. Rev. B56 (1997) 8651-8673. 
[14] Hatano N, Nelson D R: Non-Hermitian delocalization and eigenfunctions. Phys. Rev. B58 (1998) 8384-8390.

[15] Krein M G: Integral equations on a half-line with kernel depending upon the difference of the arguments. Amer. Math. Soc. Transl. 22 (1963) 163-288.

[16] Lancaster P, Tismenetsky M: The Theory of Matrices, second edition. Acad. Press, New York, 1985.

[17] Nelson D R, Shnerb N M: Non-Hermitian localization and population biology. Phys. Rev. E58 (1998) 1383-1403.

[18] Reddy S C: Pseudospectra of Wiener-Hopf integral operators and constant coefficient differential operators. J. Int. Eqns. and Applic. 5 (1993) 369-403.

[19] Reichel L, Trefethen L N: Eigenvalues and pseudoeigenvalues of Toeplitz matrices. Linear Alg. and its Applic. 162-4 (1992) 153-185.

[20] Trefethen L N: Pseudospectra of matrices. In Numerical Analysis 1991 (ed. D. F. Griffiths and G. A. Watson) pp 234-266. Harlow, UK: Longman Sci. Tech. Publ., 1992.

[21] Trefethen L N: Pseudospectra of linear operators. SIAM Review 39 (1997) 383-406.

[22] Trefethen L N: Unpublished communications.

[23] Watkins D S: Fundamentals of Matrix Computations. J Wiley and Sons, New York, 1991.

[24] Williamson J H: The Algebraic Eigenvalue Problem. Oxford Univ. Press, London, 1965.

[25] Viswanath D, Trefethen L N: Condition numbers of random triangular matrices. Preprint, 1998.

Department of Mathematics

King's College

Strand

London WC2R 2LS

England

e-mail: E.Brian.Davies@kcl.ac.uk 